\title{
Jackson-Weiss Syndrome
}

National Cancer Institute

\section{Source}

National Cancer Institute. Jackson-Weiss Syndrome. NCI Thesaurus. Code C123814.

A rare, autosomal dominant inherited disorder caused by mutations in the FGFR2 gene. It is characterized by the premature fusion of the bones of the skull (craniosynostosis) and foot abnormalities. The craniosynostosis results in a malformed skull, widely spaced eyes, and a bulging forehead. The foot abnormalities consist of short and wide first toes, which bend away from the other toes. In addition, syndactyly in some toes may be present. The hands are almost always normal. 